\title{
Deployment Control of a Tethered Satellite System for Rendezvous with a Target*
}

\author{
Hirohisa $\mathrm{KOJIMA}^{\dagger}$ and Yutaka TARUOKA
}

Department of Aerospace Engineering, Tokyo Metropolitan University, Hino, Tokyo 191-0065, Japan

\begin{abstract}
This paper considers deployment control of a tethered satellite system (TSS) for rendezvousing a subsatellite with a target object orbiting at a lower altitude. Contrary to past studies that considered a linear quadratic regulator (LQR), in the present study, an LQR-based controller is designed using a pole assignment shift technique, in which the acceleration of the tether deployment or retrieval is treated as the control input and no propellant is used. For simplicity, the TSS is represented as a dumbbell model. The tether tension is calculated based on the tether state variables (tether length, tether length rate, tether angle, tether angle rate, and acceleration of the tether deployment treated as the control input) in order to confirm whether or not tether slack occurs. The experimental results show that the proposed control law contributes to reducing the difference between the desired and experimental tether librational motions.
\end{abstract}

Key Words: Libration Motion, Tethered Satellite System, Debris Capture, Linear Quadratic Regulator

\section{Nomenclature}

$\boldsymbol{A}, \boldsymbol{B}:$ System matrix

$c$ : constant parameter for prescribing reference tether deployment speed

$\boldsymbol{F}_{0}, \boldsymbol{F}\left(\gamma_{1}\right), \boldsymbol{F}\left(\gamma_{2}\right)$ : control gain vector

I: unity matrix

$l$ : tether length, $\mathrm{km}$

$l_{0}$ : initial tether length

$m_{1}$ : mother satellite mass, $10,000 \mathrm{~kg}$

$m_{2}$ : subsatellite mass, $100 \mathrm{~kg}$

$\boldsymbol{P}$ : symmetric positive definite solution of the Riccati equation

$Q$ : symmetric positive semi-definite weighting matrix

$R$ : symmetric positive definite weighting matrix

$r_{M}$ : orbital radius of mother satellite, $\mathrm{km}$

$r_{T}$ : orbital radius of target satellite, $\mathrm{km}$

$T$ : tether tension, $\mathrm{N}$

$t_{1}, t_{2}$ : switched timing of tether deployment sequence

$t^{*}$ : orbital period, $\mathrm{s}$

$u$ : feedback control

$v_{s}$ : orbital velocity of subsatellite, $\mathrm{km} / \mathrm{s}$

$v_{T}$ : orbital velocity of target satellite, $\mathrm{km} / \mathrm{s}$

$\boldsymbol{x}$ : state vector, $\boldsymbol{x}=\left[\begin{array}{lll}\Delta \theta & \Delta \dot{\theta} \Delta l \Delta \dot{l}^{\mathrm{T}}\end{array}\right.$

$\theta$ : tether pitch angle, rad

$\omega_{M}$ : orbital angular velocity of mother satellite, $\mathrm{rad} / \mathrm{s}$

$\omega_{T}$ : orbital angular velocity of target satellite, $\mathrm{rad} / \mathrm{s}$

$\gamma_{1}, \gamma_{2}:$ pole assignment

$\mu$ : Earth gravitational constant, $3.9861 \times 10^{5} \mathrm{~km}^{3} / \mathrm{s}^{2}$

$\phi$ : inclination angle of slope

$\dot{\eta}$ : rotational angular speed of experimental setup, $\mathrm{rad} / \mathrm{s}$

(C) 2016 The Japan Society for Aeronautical and Space Sciences *Received 2 June 2015; final revision received 27 June 2016; accepted for publication 20 July 2016

†Corresponding author, hkojima@tmu.ac.jp
Subscripts

$d$ : reference libration parameter

( ) : differentiation with respect to time

\section{Introduction}

A tethered satellite system (TSS) is a large flexible structure composed of a number of satellites connected by space tethers. The flexibility, high strength, light weight, and long length are advantages of the TSS, which can also reduce fuel consumption. Because of these advantages, a number of TSSs have been proposed for various future applications. ${ }^{1)}$

One promising application of the TSS is a sky hook for capturing a payload orbiting in low Earth orbit (LEO). ${ }^{2,3)}$ In this paper, we investigate the deployment control of a TSS. The TSS rendezvous problem is very important, because if a tether is deployed upward, this application can be used as a debris capture and removal system, ${ }^{4)}$ and an optimal control strategy can be used. ${ }^{5-8)}$ However, optimal control requires extensive calculation time to obtain optimal solutions, and because it is feedforward control, it is not robust to unpredicted situations such as initial condition errors, modeling errors and external disturbances. As feedback control, model predictive control (MPC) with ProportionalDerivative (PD) tension control was presented for the rendezvous maneuvering in the orbital plane. ${ }^{9-11)}$ The control algorithm for MPC consists of two control loops. One is intended to control the width of the tether swing, and the other is intended to control the swing time of the tether toward the rendezvous point. MPC is effective for controlling nonlinear systems. However, since MPC is a computationally intensive task, its applicability to real space missions is still not easy. Extension of the time available for rendezvousing by adding an actuator moving up and down on the tether in order to increase the degree of freedom has also been investigated. ${ }^{9-11)}$ However, an additional movable mass on the tether makes 
the dynamics more complex. Instead of an additional movable mass, tether tension and thrusters can be used to control the tether swing. ${ }^{12,13)}$ However, any movable mass on the tether and thrusters obviously results in an increase in the mass of the TSS. In a previous study, ${ }^{14)}$ linear feedback of the tether length and tether length rate of change was shown to be sufficient to guarantee the asymptotic stability of a closed-loop system to a desired equilibrium point under the assumption of no mass and a non-flexible tether. However, since it is not easy to carry out real space missions to validate controllers, few missions have been performed to experimentally verify linear feedback control of the tether length and its rate of change (e.g., Ref. 14)). Furthermore, the controller proposed in the previous study ${ }^{14)}$ was intended to keep the final librational angle amplitude within a specified range, but was not intended for use in controlling the rendezvous between a subsatellite and an orbiting target object.

As a candidate solution to overcome the above problems, in this paper, we consider an LQR-based control method cooperating with a pole assignment shift technique ${ }^{15)}$ to make the controller more robust to perturbations than a simple LQR-based controller. In addition, in contrast to a previous study, ${ }^{13)}$ in which the tether tension and thruster were assumed as control inputs, in this paper, we assume tether deployment control without a thruster. Furthermore, we intend to verify the validity of the controller not only numerically, but also experimentally using a ground-based TSS motion emulator, ${ }^{16-18)}$ in which the slope inclination angle is changed to emulate the gravity gradient affecting the subsatellite as much as possible. The experimental results show that the libration motion is successfully emulated on the ground-based TSS motion emulator and that the control law contributes to reduce the difference between the desired and experimental tether librational motions.

The remainder of this paper is organized as follows. In Section 2, the TSS model assumed is described. The controller design and the results of numerical simulations are presented in Section 3. In Section 4, the experimental setup is described and the experimental results are presented. Finally, a conclusion is presented in Section 5.

\section{System Model Description}

\subsection{Assumptions}

Although a number of previous studies assumed a flexible and massive tether (e.g. Ref. 19)), for simplicity, in this study, we use a dumbbell-shaped TSS, as shown in Fig. 1. This model comprises two satellites: the mother satellite and a subsatellite. The following assumptions are made in deriving the equations of motion for this model.

1) The mother satellite (main spacecraft) has sufficient mass so that the center of mass of the system is coincident with the position of the mother satellite.

2) The mother satellite is in an unperturbed circular orbit at an altitude of $500 \mathrm{~km}$.

3) Only the in-plane motion of the system is considered.

4) The satellites are considered to be point masses.

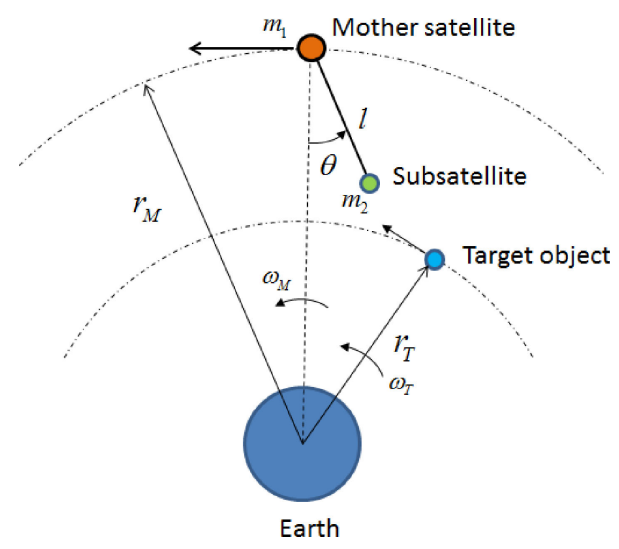

Fig. 1. System model of the TSS.

5) The tether mass and flexibility are neglected.

6) No external force is considered other than Earth's gravity.

Generally speaking, as a result of the shift of the mass distribution resulting from a change in the tether length, the altitude of the mother satellite will change, and the orbital motion will shift. In addition, the out-of-plane motion and disturbances should be taken into consideration in order to capture a payload in space. However, in this study, we neglect these effects in order to simplify the problem.

\subsection{Equations of motion}

The equations of motion governing a dumbbell-shaped TSS can be obtained using the Lagrange equation under the assumptions made in this study, as follows ${ }^{7}$ :

$$
\left\{\begin{array}{l}
\ddot{l}-l \dot{\theta}\left(\dot{\theta}+2 \omega_{M}\right)-3 \omega_{M}{ }^{2} l \cos ^{2} \theta=-T / m_{2} \\
\ddot{\theta}+2\left(\dot{\theta}+\omega_{M}\right)(\dot{l} / l)+3 \omega_{M}{ }^{2} \cos \theta \sin \theta=0
\end{array}\right.
$$

In these equations, the libration is affected by the gravity gradient and the Coriolis force which depends on the tether deployment or retrieval velocity. Therefore, the libration can be controlled by a control input depending on the tether length, the tether deployment or retrieval velocity, and the libration angle.

\section{Tether Deployment Control}

\subsection{Feedforward control}

If we want to capture a target orbiting in a lower orbit using a TSS, we need to make the subsatellite rendezvous with the target. We should also control the subsatellite to have the same velocity as that of the target at the same altitude. The deployment process must be chosen carefully in order to smoothly decrease the tether deployment speed using the tether tension. To this end, we consider the following tether deployment sequence ${ }^{20)}$ :

$$
\left\{\begin{array}{l}
l=l_{0} \exp \left(\frac{c t}{t^{*}}\right) \quad(1.0[\mathrm{~km}] \leq l<30[\mathrm{~km}]) \\
l=30\left(\frac{c}{t^{*}}\right)\left(t-t_{1}\right)+30 \quad(30[\mathrm{~km}] \leq l<70[\mathrm{~km}]) \\
l=100-30 \exp \left\{-\frac{c\left(t-t_{2}\right)}{t^{*}}\right\}(70[\mathrm{~km}] \leq l \leq 100[\mathrm{~km}])
\end{array}\right.
$$


The deployment velocity is divided into three phases because it is natural to have acceleration, constant-velocity, and decelerating phases for tether length control. The parameter $c$ is an arbitrary constant. The deployment velocity can be controlled by changing this parameter.

Figure 2 shows the results of the numerical simulations for the case in which $c=4.83$ and the initial tether swing angle and tether swing angular velocity are $0 \mathrm{rad}$ and $0 \mathrm{rad} / \mathrm{s}$, respectively. Figure 2(e) shows the subsatellite trajectory in the local vertical and local horizontal (LVLH) coordinate system. Figures 2(a), 2(b), and 2(e) indicate that libration is induced. The swing angle is in the range from $-0.296 \mathrm{rad}$ to $+0.296 \mathrm{rad}$, and the angular velocity is in the range from $-5.6 \times 10^{-4} \mathrm{rad} / \mathrm{s}$ to $+5.6 \times 10^{-4} \mathrm{rad} / \mathrm{s}$ after the end of tether deployment. The libration period is ap- proximately $3,300 \mathrm{~s}$. Figures $2(\mathrm{c})$ and $2(\mathrm{~d})$ indicate that it takes $12,122 \mathrm{~s}$ to deploy the tether from $1.0 \mathrm{~km}$ to $100 \mathrm{~km}$. Figure 2(f) indicates that the subsatellite velocity matches the velocity of the target at a tether angle of $0 \mathrm{rad}$ and an altitude of $400 \mathrm{~km}$. Therefore, this libration can be used as a reference for rendezvousing with the target.

\subsection{Linear feedback libration control}

In order to reduce errors between the reference libration and the actual libration to zero, we design a linear feedback controller using pole assignment shift techniques. We consider the differences between the real and reference values of the tether angle, angular velocity, length, and deployment or retrieval velocity as the errors. In the present study, the tether length acceleration is considered as a virtual control input. The tether tension is then calculated using the equations

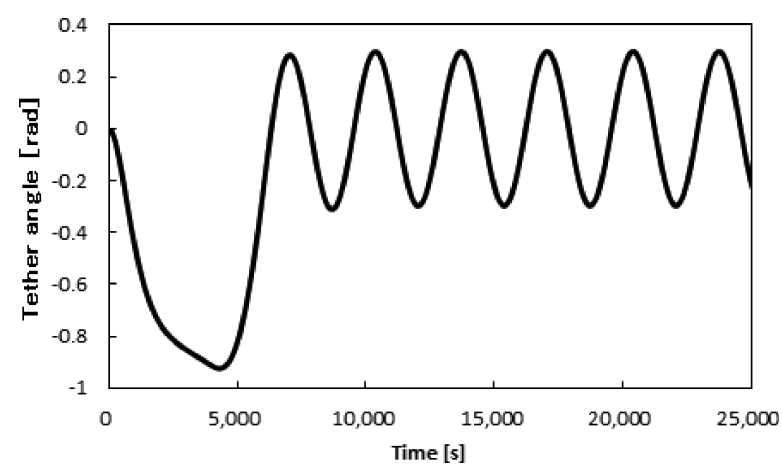

(a) Tether angle

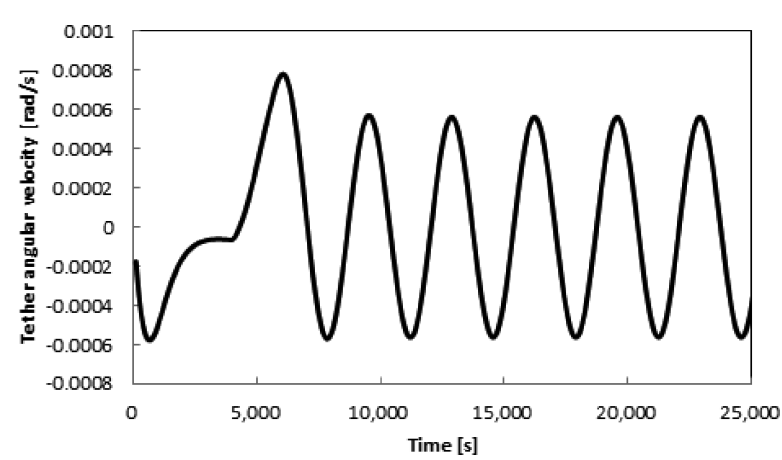

(b) Tether angular velocity

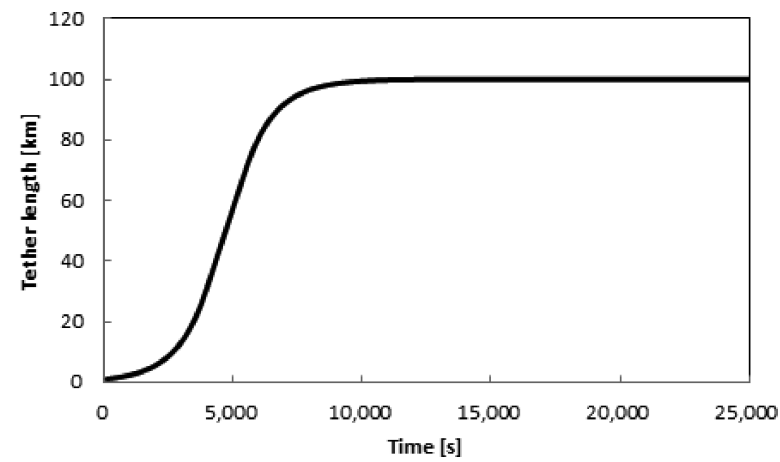

(c) Tether length

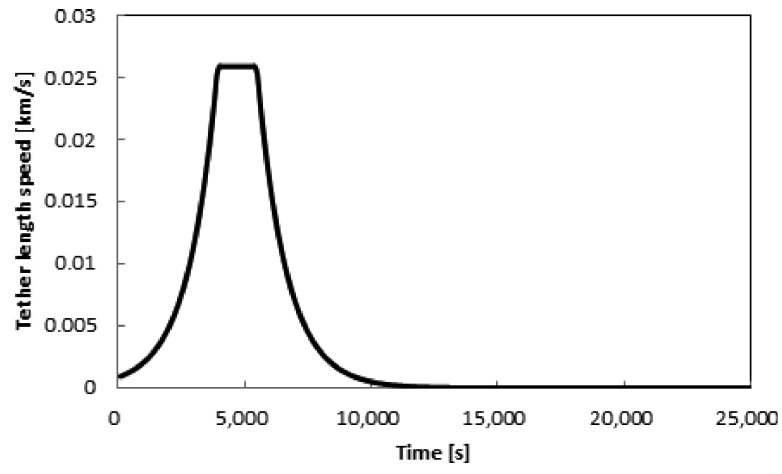

(d) Tether length speed

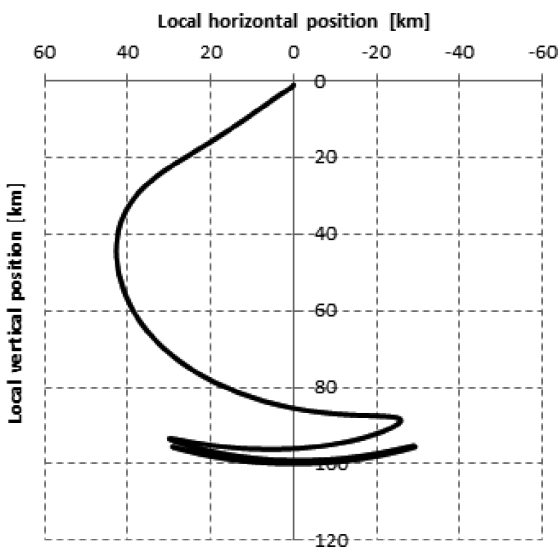

(e) Subsatellite trajectory

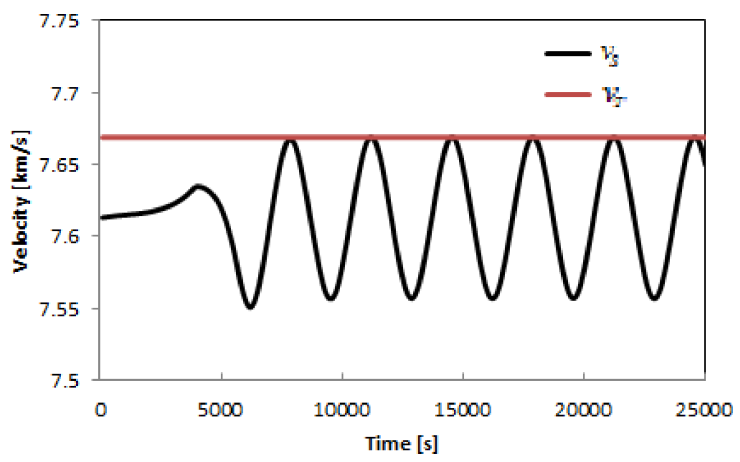

(f) Velocity of subsatellite and target object

Fig. 2. Results of numerical simulation for the feedforward deployment procedure. 
of motion, given by Eq. (1).

First, suppose that the errors are given by Eq. (3) at the target rendezvous point $\left(\theta_{d}=0.0 \mathrm{rad}, l_{d}=100 \mathrm{~km}\right)$

$$
\left\{\begin{array}{l}
\theta=\theta_{d}+\Delta \theta \\
\dot{\theta}=\dot{\theta}_{d}+\Delta \dot{\theta} \\
l=l_{d}+\Delta l \\
i=\dot{l}_{d}+\Delta \dot{l} \\
T=T_{d}+\Delta T
\end{array}\right.
$$

where $\dot{\theta}_{d}$ is determined as $\dot{\theta}_{d}=\left(r_{T} \omega_{T}-r_{M} \omega_{M}\right) / l_{d}$ to rendezvous with the target satellite.

By substituting Eq. (3) into Eq. (1), the linearized equations of Eq. (1) around the reference libration at the target rendezvous point are obtained as follows:

$\left\{\begin{array}{l}\Delta \ddot{l}=200\left(a+\omega_{M}\right) \Delta \dot{\theta}+\left(a^{2}+2 a \omega_{M}+3 \omega_{M}^{2}\right) \Delta l-\frac{\Delta T}{m_{2}} \\ \Delta \ddot{\theta}=-\frac{a+\omega_{M}}{50} \Delta \dot{l}-3 \omega_{M}^{2} \Delta \theta\end{array}\right.$

where $a$ is $-5.6 \times 10^{-4}$, which is the value of the tether angular velocity $\dot{\theta}_{d}$ at the target rendezvous point.

By representing the state vector as a set of deviations from the reference libration as

$$
\boldsymbol{x}=\left[\Delta \theta \Delta \dot{\theta} \Delta l \Delta \dot{l}^{\mathrm{T}}\right.
$$

the linearized equation can be rewritten in the state space form by treating acceleration of the tether length, instead of tension error, as the control input, as follows:

$$
\begin{aligned}
\dot{\boldsymbol{x}} & =\left[\begin{array}{cccc}
0 & 1 & 0 & 0 \\
-3 \omega_{M}{ }^{2} & 0 & 0 & -\frac{a+\omega_{M}}{50} \\
0 & 0 & 0 & 1 \\
0 & 0 & 0 & 0
\end{array}\right] \boldsymbol{x}+\left[\begin{array}{l}
0 \\
0 \\
0 \\
1
\end{array}\right] u \\
& =\boldsymbol{A} \boldsymbol{x}+\boldsymbol{B} u \\
y & =\left[\begin{array}{llll}
1 & 0 & 0 & 0
\end{array}\right] \boldsymbol{x}
\end{aligned}
$$

and the linear feedback control is represented as follows:

$$
u=\Delta \ddot{l}=-\boldsymbol{F} \boldsymbol{x}
$$

where $\boldsymbol{F}$ is a control gain vector. The reason why the acceleration of the tether length is treated as the control input, instead of tether tension, is that we intend to compare the numerical simulation results and experimental ones. This is done because the tether length speed determined by integrating the acceleration of the tether length can be more easily implemented in the experimental setup than the tether tension.

The control gain vector is determined from the following linear quadratic regulator problem:

"Find a control gain vector $\boldsymbol{F}$ such that the quadratic cost

\begin{tabular}{|c|c|c|c|c|c|}
\hline $\begin{array}{c}\text { Control } \\
\text { gain } \\
\boldsymbol{F}\end{array}$ & $\begin{array}{c}\text { Pole } \\
\text { assignment } \\
\gamma\end{array}$ & $F_{1}$ & $F_{2}$ & $F_{3}$ & $F_{4}$ \\
\hline $\boldsymbol{F}_{0}$ & - & -7.7460 & -8.6887 & 7.7460 & 8.6886 \\
\hline $\boldsymbol{F}\left(\gamma_{1}\right)$ & $1.24 \times 10^{-4}$ & -350.38 & $1,138.8$ & 7.8764 & 8.6894 \\
\hline $\boldsymbol{F}\left(\gamma_{2}\right)$ & $2.88 \times 10^{-4}$ & -813.61 & 62,596 & 8.4455 & 8.6903 \\
\hline
\end{tabular}
function is minimized:

$$
J=\int_{0}^{\infty}\left(\boldsymbol{x}^{\mathrm{T}} \boldsymbol{Q} \boldsymbol{x}+R u^{2}\right) \mathrm{d} t
$$

where $\boldsymbol{Q}$ is a symmetric positive semi-definite weighting ma-
Table 1. Control gains.

trix and $R$ is a symmetric positive definite weighting matrix." Setting weighting matrices $Q$ and $R$ for LQR as $\operatorname{diag}(60,60,60,60)$ and 1 , respectively, the control gain vector $\boldsymbol{F}$ for the LQR can be obtained as listed in Table 1.

The poles obtained without using the pole assignment shift technique, i.e., for $\boldsymbol{F}_{0}$, are pure imaginary numbers. This implies that the state errors for the case of $\boldsymbol{F}_{0}$ will never converge to zero. Accordingly, we redesigned control gains $\boldsymbol{F}\left(\gamma_{1}\right)$ and $\boldsymbol{F}\left(\gamma_{2}\right)$ using the pole assignment shift technique. ${ }^{15)}$ We chose small values for $\gamma$ as the pole assignment shift because, if a large value is chosen for $\gamma$, the tether tension is likely to become too large, or even negative. The LQR control design with and without using the pole assignment shift technique $^{15)}$ is briefly explained in the Appendix.

\subsubsection{Tether tension limitation}

The tether tension must not be less than $0 \mathrm{~N}$ because a negative tether tension means that the tether pushes the subsatellite. The tether can pull, but cannot push, the subsatellite. In addition, there is a risk of the tether breaking in the case of high tether tension. We assume that the tether material is Kevlar with an ultimate tensile strength of 3,000 MPa, and that the tether cross-section area is $0.167 \mathrm{~mm}^{2}$. From these values, we chose the upper bound of $5,000 \mathrm{~N}$ for the tether tension. Therefore, we place a constraint on the tether tension so that it is limited to within the range of 0 to $5,000 \mathrm{~N}$. If the tension is under $0 \mathrm{~N}$ or over $5,000 \mathrm{~N}$, we check the errors of the tether state variables from the reference and determine the virtual control input (tether length acceleration/deceleration) so that the tether tension will not exceed the limitation.

\subsubsection{Results of numerical simulations}

We assume that at the target rendezvous point

$$
\left(\theta_{d}, \dot{\theta}_{d}, l_{d}, \dot{l}_{d}\right)=(0,-0.00056,100,0)
$$

the following errors occur

$$
(\Delta \theta, \Delta \dot{\theta}, \Delta l, \Delta \dot{l})=\left( \pm 0.002, \pm 5.0 \times 10^{-6}, \pm 0.05, \pm 0.05\right)
$$

Numerical simulations are carried out for the above errors in order to verify the validity of the controllers. Figure 3 shows the results of the numerical simulations and whether or not the errors converge to zero. Here, we used the control gain $\boldsymbol{F}\left(\gamma_{2}\right)$, and the error conditions $(\Delta \theta, \Delta \dot{\theta}, \Delta l, \Delta \dot{l})$ are $\left(0.002 \mathrm{rad},-5.0 \times 10^{-6} \mathrm{rad} / \mathrm{s},-0.05 \mathrm{~km},-0.05 \mathrm{~km} / \mathrm{s}\right)$. As can be seen from the results, the errors converged to zero and the convergence time was 5,944s.

The time history of the tether tension with the tether tension limitation is shown in Fig. 3(e). Figure 3(e) indicates that the feedback control is performed successfully and the tether tension is successfully limited within the range of 0 to $5,000 \mathrm{~N}$. 


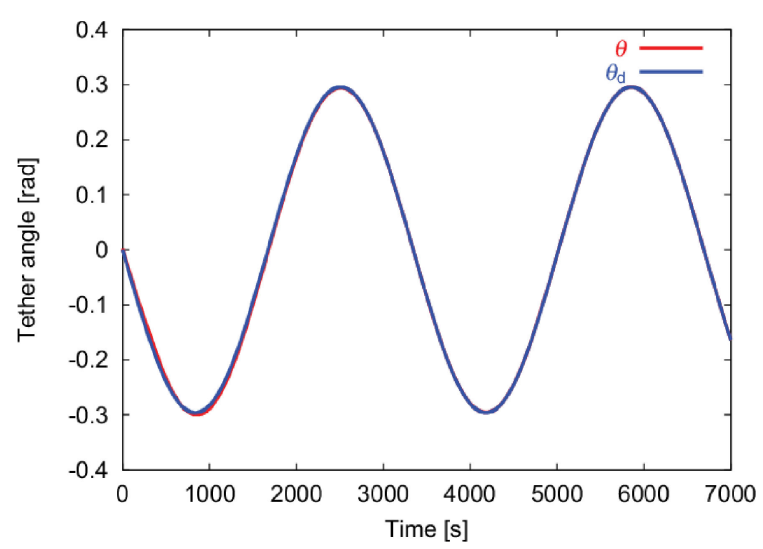

(a) Tether angle

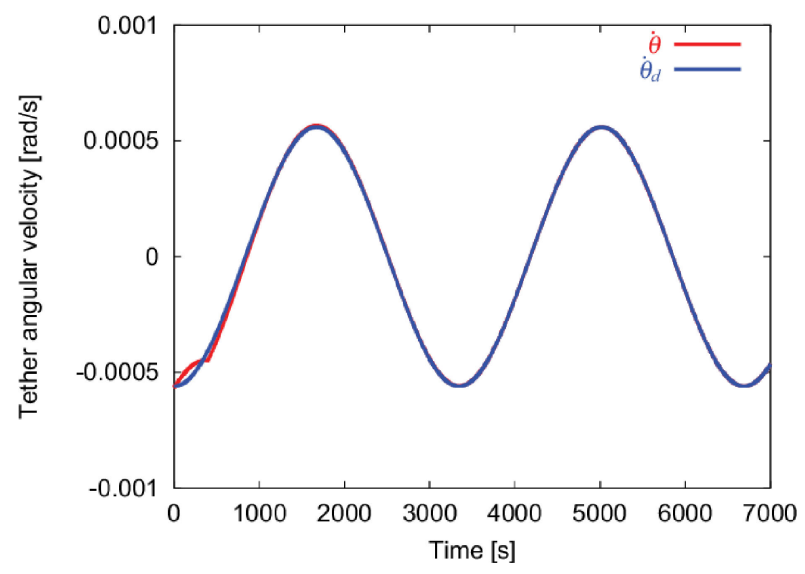

(b) Tether angular velocity

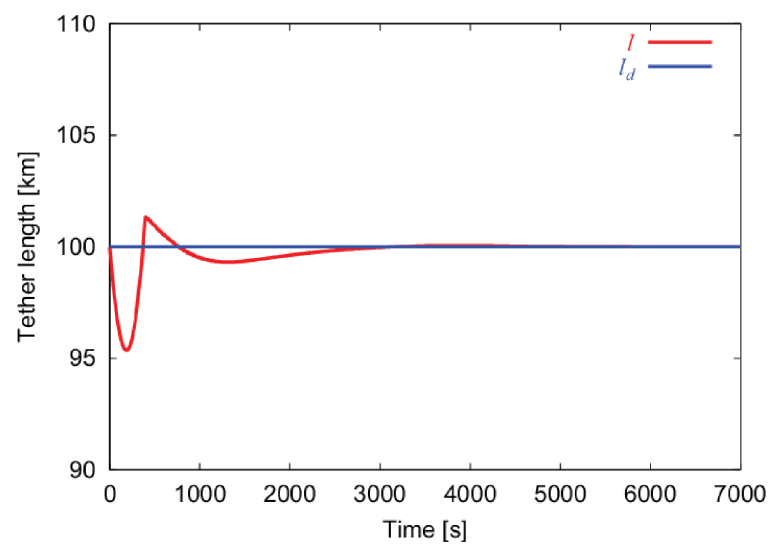

(c) Tether length

\subsubsection{Convergence time}

The convergence time is defined as the time until the absolute tether length error with respect to the reference length becomes less than $4 \mathrm{~m}$ and the absolute tether swing angle error becomes less than 0.002 rad.

The convergence times for the initial error conditions are listed in Table 2 and shown in Fig. 4.

The convergence time for the case of $\boldsymbol{F}\left(\gamma_{2}\right)$ is less than that for the case of $\boldsymbol{F}\left(\gamma_{1}\right)$, except for cases 9, 10 and 11. Although control gain $\boldsymbol{F}\left(\gamma_{2}\right)$ is designed with larger $\gamma$ than $\boldsymbol{F}\left(\gamma_{1}\right)$, the convergence time for $\boldsymbol{F}\left(\gamma_{2}\right)$ is greater than that for $\boldsymbol{F}\left(\gamma_{1}\right)$ for cases 9,10 and 11 . The reason for these unexpected results may be explained as follows. In cases 9 and 10, the

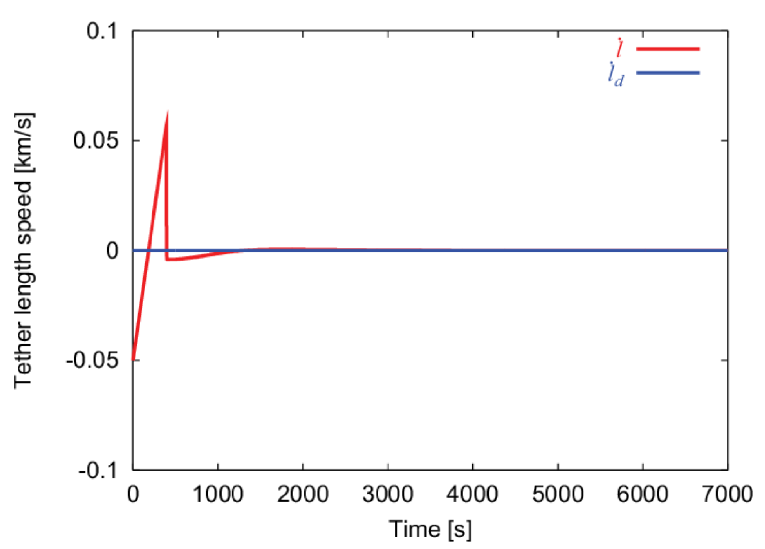

(d) Tether length speed

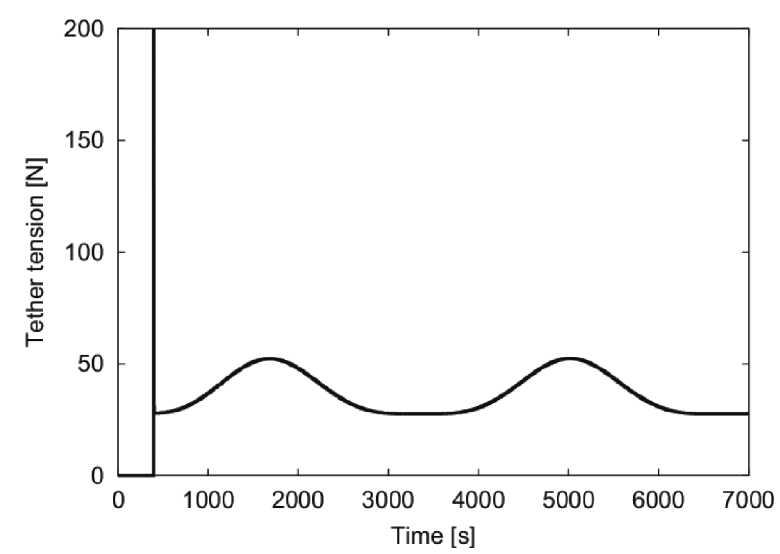

(e) Tether tension

Fig. 3. Numerical results of feedback control

tether angle and angular velocity are less than that of the reference states, and the tether length is longer than that of the reference length. In such cases, the feedback controller determines the tether length acceleration that results in reducing the tether length and boosting the tether angular velocity due to the Coriolis force, that it, the subsatellite is pulled towards the mother satellite. Because the control gain $\boldsymbol{F}\left(\gamma_{2}\right)$ is designed with larger $\gamma$ than $\boldsymbol{F}\left(\gamma_{1}\right)$, the resulting tension determined by $\boldsymbol{F}\left(\gamma_{2}\right)$ becomes larger than that of $\boldsymbol{F}\left(\gamma_{1}\right)$. As the result, the tether becomes slack and the state of TSS does not reach the reference state for $\boldsymbol{F}\left(\gamma_{2}\right)$ in cases 9 and 10 .

On the other hand, the convergence time for the case of $\boldsymbol{F}\left(\gamma_{2}\right)$ is almost half of that for $\boldsymbol{F}\left(\gamma_{1}\right)$ in cases 1 to 8 . There- 
Table 2. Convergence time for feedback control.

\begin{tabular}{|c|c|c|c|c|c|c|}
\hline \multirow{2}{*}{ Case No. } & \multirow{2}{*}{$\Delta \theta$} & \multirow{2}{*}{$\Delta \dot{\theta}$} & \multirow{2}{*}{$\Delta l$} & \multirow{2}{*}{$\Delta i$} & \multicolumn{2}{|c|}{ Convergence time } \\
\hline & & & & & $\boldsymbol{F}\left(\gamma_{1}\right)$ & $\boldsymbol{F}\left(\gamma_{2}\right)$ \\
\hline 1 & + & + & + & + & $6,434.897$ & $2,491.91$ \\
\hline 2 & + & + & + & - & $10,610.96$ & $5,509.37$ \\
\hline 3 & + & + & - & + & $6,228.22$ & $2,196.90$ \\
\hline 4 & + & + & - & - & $10,704.74$ & $5,530.63$ \\
\hline 5 & + & - & + & + & $7,053.08$ & $4,563.52$ \\
\hline 6 & + & - & + & - & $10,682.67$ & $5,550.37$ \\
\hline 7 & + & - & - & + & $8,324.11$ & $4,625.32$ \\
\hline 8 & + & - & - & - & $10,707.53$ & $5,569.37$ \\
\hline 9 & - & + & + & + & $14,581.49$ & - \\
\hline 10 & - & + & + & - & $14,587.07$ & - \\
\hline 11 & - & + & - & + & $7,806.95$ & $8,320.67$ \\
\hline 12 & - & + & - & - & $9,249.32$ & $8,331.59$ \\
\hline 13 & - & - & + & + & $14,509.75$ & $8,806.43$ \\
\hline 14 & - & - & + & - & $14,490.51$ & $9,154.96$ \\
\hline 15 & - & - & - & + & $8,884.01$ & $7,202.05$ \\
\hline 16 & - & - & - & - & $10,445.80$ & $7,148.39$ \\
\hline
\end{tabular}

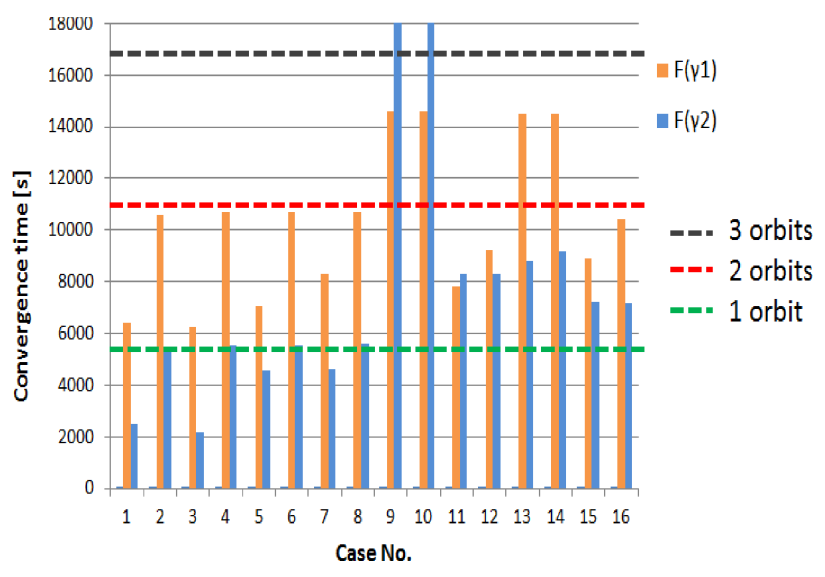

Fig. 4. Convergence time of feedback control.

fore, it is desirable to use higher gain control for cases when the tether angle is greater than the reference angle.

The orbital period of the TSS is 5,589 s. The convergence time for the case of $\boldsymbol{F}\left(\gamma_{2}\right)$ is approximately 0.446 to 1.64 orbital periods, except for cases 9,10 and 11 . The risk of failure increases as the time required for control increases. The resulting convergence time may still be too long when the proposed controller is simply applied to the TSS. Furthermore, no external disturbances are taken into account in this study. Therefore, further improvement will be required.

\section{Experimental Results for Feedforward Control}

\subsection{Experimental setup}

We used the experimental setup shown in Fig. 5 to investigate the feedback control. ${ }^{16-18)}$

This setup consists of the following three stages:

1) Rotation stage: The orbital velocity of the satellite can be reproduced by rotating this stage.

2) Slider stage: The orbital radius change of the mother satellite can be reproduced by shifting the slider stage equipped with the reel mechanism.

3) Slope: The gravitational force can be reproduced by

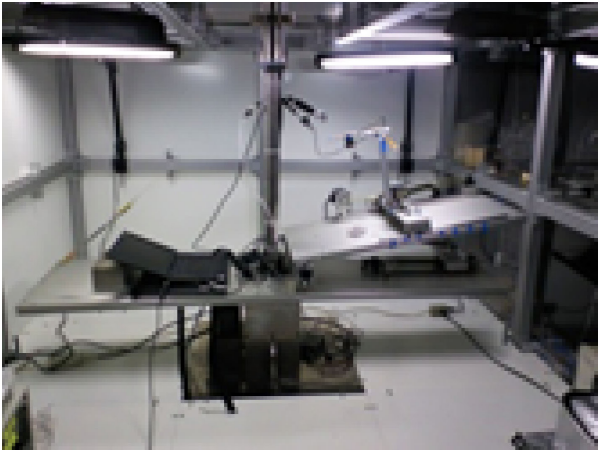

Fig. 5. Experimental apparatus.

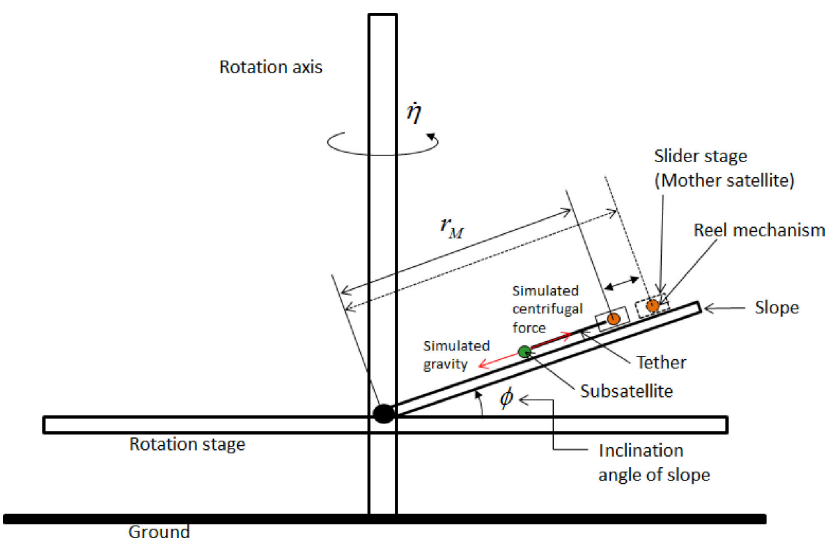

Fig. 6. Schematic representation of the main component of the experimental setup.

changing the inclination angle of the slope.

Figure 6 shows a schematic representation of the main component of the experimental setup. The simulated centripetal and centrifugal forces are reproduced by the force affecting the satellite model in the slope direction.

By synchronizing the motion of each motor, the desired TSS trajectory can be reproduced. Since stepper motors are used for the stages, we can execute precise speed control. This setup deploys the tether using a reel mechanism. By placing dry ice under the subsatellite model, the friction between the subsatellite model and the slope can be eliminated to the greatest extent possible.

Figure 7 shows a block diagram of the relationship among a sensor, actuator and computers. The sensing and control procedure is explained as follows:

A small red marker is installed on the subsatellite model. A charge coupled device (CCD) camera is installed above the slope to detect the position of the subsatellite model from the image taken by the CCD camera. Software to detect the subsatellite position is implemented in a laptop PC using $\mathrm{C}$ language OpenCV. The position of the subsatellite is sent to a desktop PC next to the experimental apparatus through a wireless LAN. The tether length and tether libration angle are determined based on the position of the subsatellite. The tether length rate of change and tether angular velocity are calculated by numerical differentiation, and a low-pass filter is utilized to reduce the noise in the tether length rate of change 


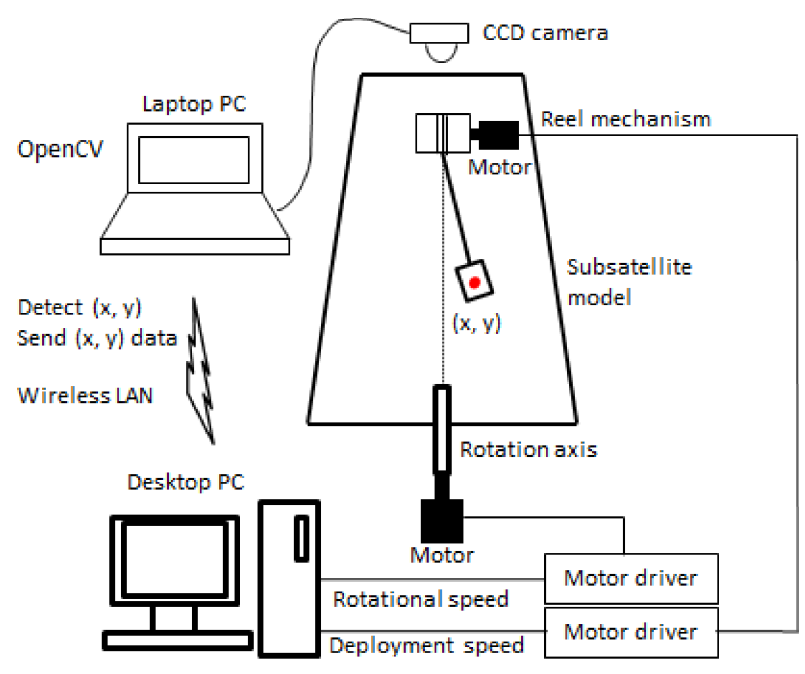

Calculate TSS state from current and previous $(x, y)$ Calculate $\ddot{L}$ based on control scheme

Integrate $\ddot{L}$ to determine $\dot{L}$ in next step

Fig. 7. Block diagram of the experimental devices.

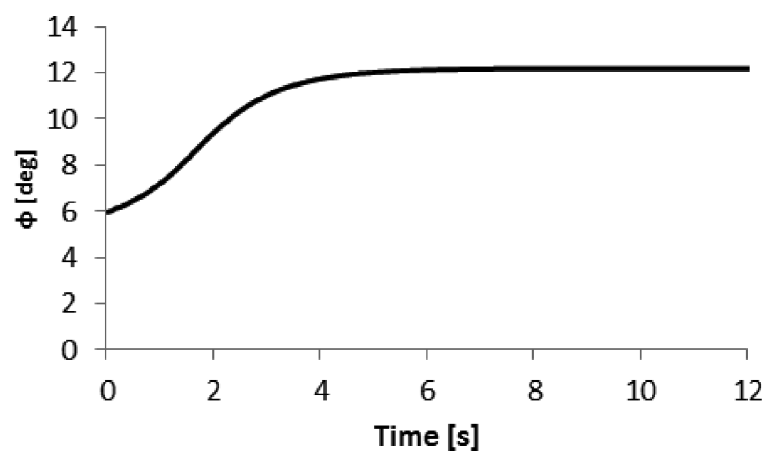

Fig. 8. Time history of the slope angle to emulate the gravity gradient.

and tether angular velocity determined by numerically differentiating the tether length and tether angle. Since the tether tension cannot be directly sensed and controlled in this setup, the command tether deployment speed is determined by the desktop PC through numerically integrating the equations of motion in accordance with the commanded acceleration of the tether length. The rotational speed of the reel mechanism is then changed in order to track the tether length rate of change.

Although the gravity gradient affecting the subsatellite changes in accordance with the subsatellite position on the slope, for simplicity, the inclination angle of the slope, $\phi$, was gradually raised from 6 to $12 \mathrm{deg}$ in accordance with the reference tether length, as shown in Fig. 8, so as to emulate the gravity gradient affecting the TSS.

\subsection{Experimental conditions}

If an experiment using an actual-scale model could be carried out on the ground, the scale conversion would not be necessary. However, it is impossible to carry out such experiments on the ground because the tether length and time scale are too long. In order to emulate the TSS motion using the ground-based experimental setup, we need to convert the scale of a real model to that of the experimental setup.
Table 3. Comparison of scales.

\begin{tabular}{lccc}
\hline Real model & $\begin{array}{c}\text { Experimental } \\
\text { model }\end{array}$ & $\begin{array}{c}\text { Scale ratios } \\
\text { of the real } \\
\text { model to } \\
\text { experimental } \\
\text { model }\end{array}$ \\
\hline $\begin{array}{l}\text { Orbital period, } t^{*} \\
\text { Tether length, } l\end{array}$ & 11.1 to $100 \mathrm{~km}$ & 4.0 to $36.0 \mathrm{~cm}$ & $2.78 \times 10^{5}$ \\
$\begin{array}{l}\text { Mass of } \\
\text { subsatellite, } m_{2}\end{array}$ & $100 \mathrm{~kg}$ & $90 \mathrm{~g}$ & $1.1 \times 10^{2}$ \\
\hline
\end{tabular}

Table 3 shows a comparison between real space and experimental scales, and the scale ratios of the real model to that of the experimental model. These scale ratios are used to convert the simulated results for the real model to that of the experimental setup so that we can compare the simulated results to the experimental results. The orbital period emulated in the experiments is $6.0 \mathrm{~s}$, that is, the rotational speed of the inclined table, $\dot{\eta}$, is $1.0472 \mathrm{rad} / \mathrm{s}$.

On the scale of the experimental setup, the tether can deploy from $4.0 \mathrm{~cm}$ to $36 \mathrm{~cm}$. On the other hand, we assumed that in the real space simulation, the tether is deployed in the range from $11.1 \mathrm{~km}$ to $100 \mathrm{~km}$. Before the experiment, we carried out a simulation to consider the correlation between the real and experimental scales. Figure 9 shows the results of the simulation for $c=5.18$. We deployed the tether from $11.1 \mathrm{~km}$ to $100 \mathrm{~km}$. As shown in Figs. 9(a) and 9(b), if the tether was deployed as shown in Figs. 9(c) and 9(d), libration would occur as expected. These results are regarded as references for the experiment. The experiment was carried out after converting the scale of Fig. 9 to that of the experimental setup. The sampling periods for sensing and control are 0.033.

\subsection{Experimental results}

The results are presented in Fig. 10. In the figure, the red and green lines correspond to the experimental results without feedback control, and the experimental results with feedback control considered, respectively. The reference results converted from those in Fig. 9 to the experimental scale are also presented in Fig. 10 as blue lines.

It is shown that the maximum librational tether angles in the experiment are quite similar to that of the numerically simulated one. This implies that Coriolis force during tether deployment is successfully emulated in the experimental setup. The experimental tether librational period after the first positive peak of tether angle is approximately $3.7 \mathrm{~s}$, which is similar to the reference tether librational period of 3.5. This implies that the gravity gradient is almost successfully emulated in the experiments.

Although libration occurs in the experiment without feedback control, as shown in Figs. 10(a) and 10(b), the tether angle and tether angular velocity at the completion of tether deployment are approximately $50 \%$ of the reference values. The main reason for the difference between the experimental results without control and the reference values may be disturbances. No effects of disturbances are taken into account in the numerical simulations during and after tether deploy- 


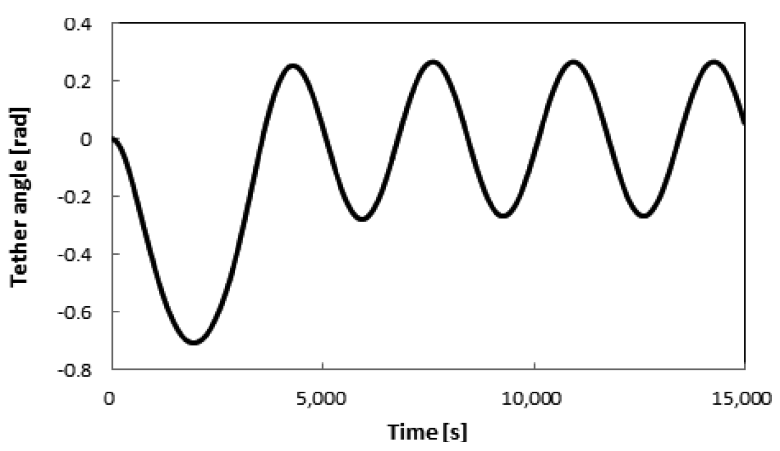

(a) Tether angle

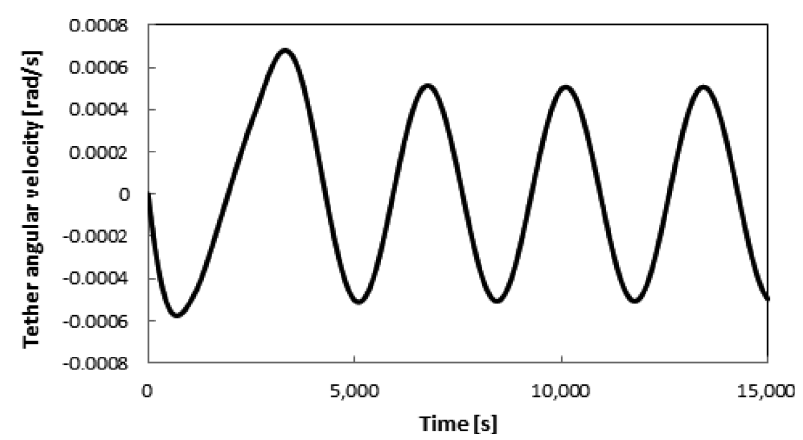

(b) Tether angular velocity

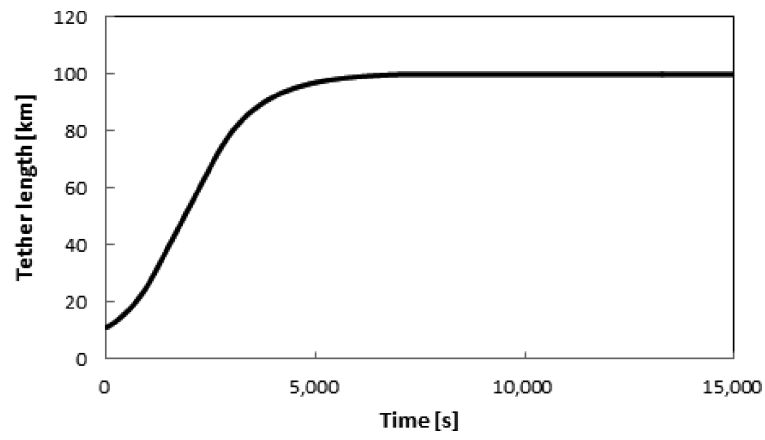

(c) Tether length

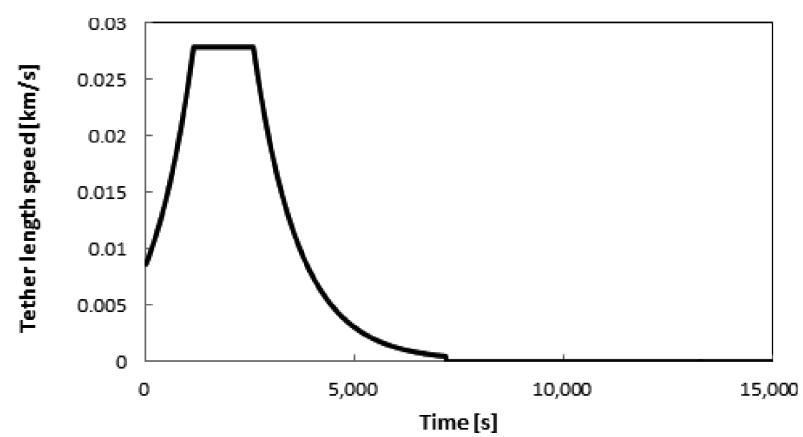

(d) Tether length speed

Fig. 9. Results of simulation.

ment. On the other hand, in the experimental environment, the subsatellite is affected by disturbances such as aerodynamic force and the frictional force between the subsatellite model and the slope. The errors may be induced by these effects.

Although the experiment without feedback control is carried out using feedforward control with the reference values, the tether deployment speed without feedback control does not track that of the references. This is because friction forces exist between the subsatellite model and the slope, and between the reel and tether. Additionally the tether deployment speed is calculated by numerically differentiating the subsatellite position measured by the CCD camera, which includes measurement noise. The finally deployed tether length is $36 \mathrm{~cm}$, which is the same length as expected, as shown in Fig. 10(c). This is because a stepper motor is used as an actuator to deploy the tether in the experiment.

The position of the subsatellite is traced by processing the image taken by the CCD camera. Noise due to the limited accuracy of image processing is included in the position of the subsatellite. The feedback control is applied $2.0 \mathrm{~s}$ after the start of deployment in order to compensate the difference between the experimental states and numerical values. It should be noted that the feedback control gains for the feedback control experiments are chosen by considering the scale factor of the experimental conditions.

It is shown in Fig. 9(d) that the tether deployment speed periodically becomes faster and slower than the reference deployment speeds in order to reduce the difference between the experimental tether angle and the reference angle by inducing the Coriolis force resulting from the change in tether deployment speed. As the result of the feedback control, the difference of tether angle between the experiment with feedback control and the reference is almost zero at the completion of tether deployment. The convergence time is approximately $5.0 \mathrm{~s}$ and corresponds to 0.833 orbits, that is, $4,658 \mathrm{~s}$ in the actual scale model. This convergence time is smaller than, but not so different from, that in the numerical simulations results.

These results show that the feedback controller in this study contributes to compensate external disturbance and to track the desired tether librational motion.

\section{Conclusion}

For the purpose of rendezvousing a tethered subsatellite with a target object orbiting in a lower orbit, feedforward and linear feedback controllers were considered. For feedforward control, we considered a tether deployment sequence consisting of three phases. For feedback control, a linear quadratic regulator was designed using a pole assignment shift technique, where we treated the tether length acceleration as a virtual control input. The controller was then numerically investigated with respect to its robustness to the initial errors from the desired librational motion. The numerical simulation results show that libration with the same velocity as a target at a rendezvous point can be achieved using feedforward control and that the errors can be made to converge to zero using feedback control.

The controller was also experimentally studied using a ground-based experimental setup intended to emulate the tether libration motion. The experimental results show that tether librational motion is successfully emulated using the ground-based experimental setup and that the feedback controller contributes to compensate external disturbance and to track the desired tether librational motion. 


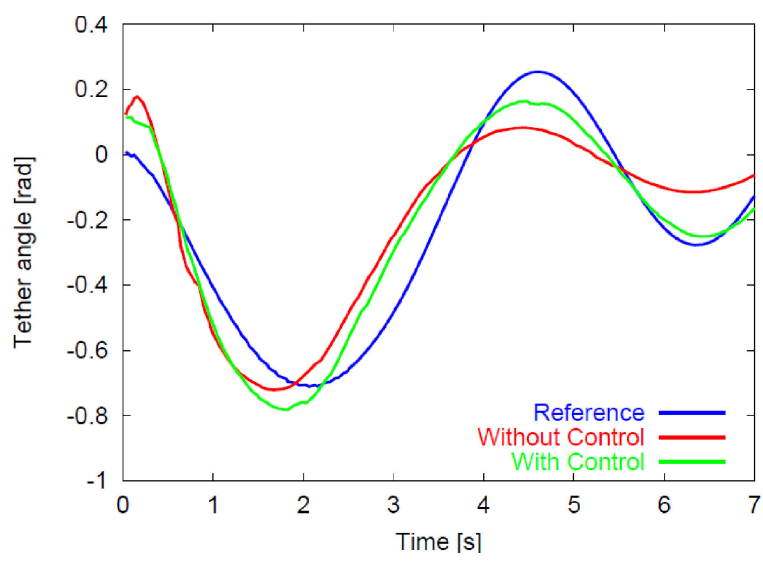

(a) Tether angle

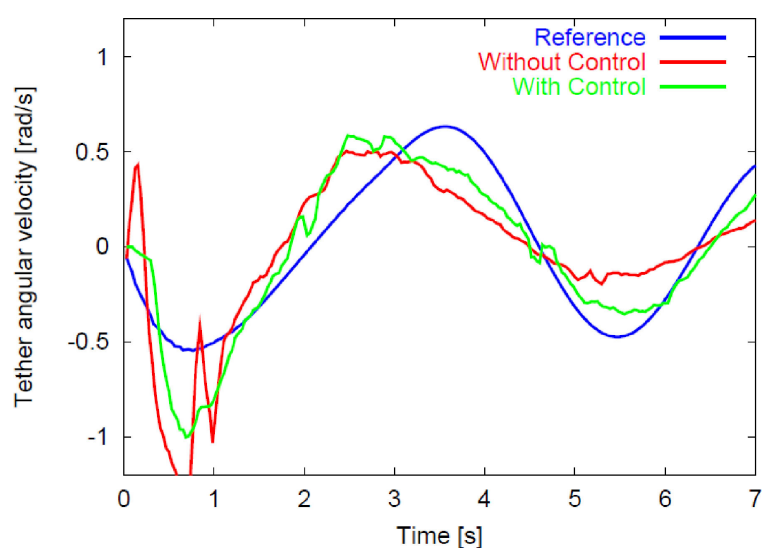

(b) Tether angular velocity

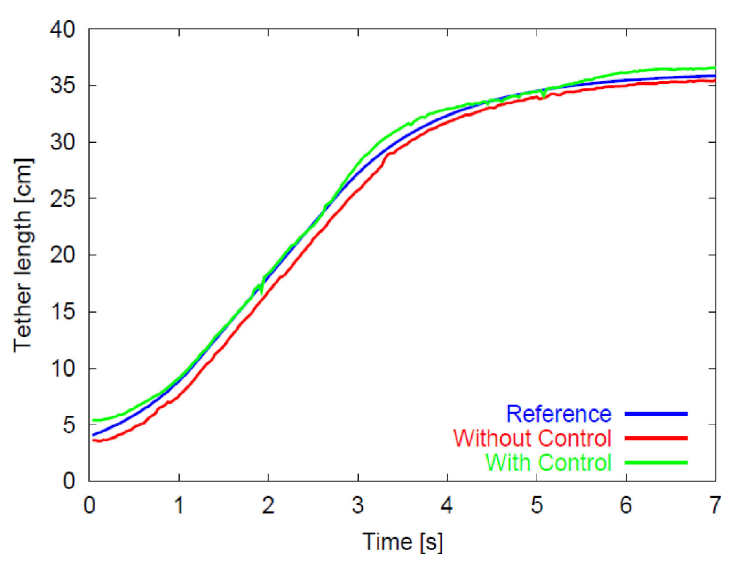

(c) Tether length

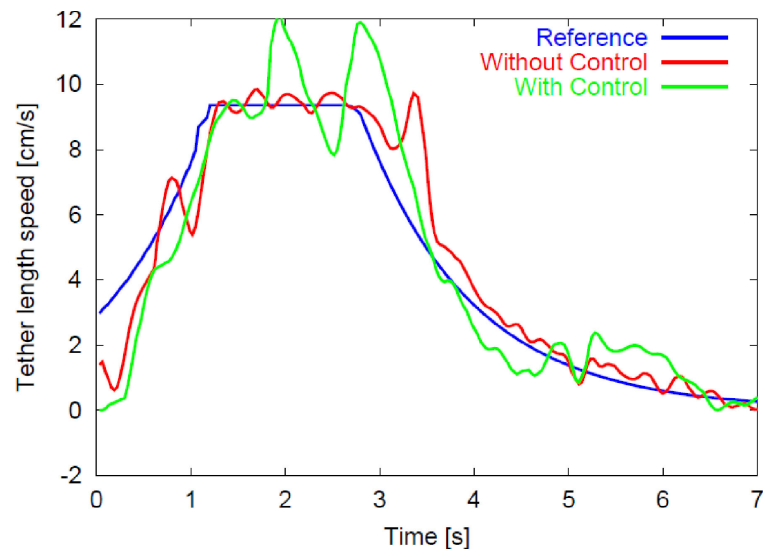

(d) Tether length speed

Fig. 10. Experimental results.

However, deploying the tether and causing the errors to converge to zero requires a significant amount of time. Originally, the motion of the TSS was strongly nonlinear, but in this paper, the motion is linearized around the reference motion. Therefore, we will need to investigate another control method to shorten the convergence time in tracking the reference librational motion by taking into account the nonlinearity and experimentally study the convergence performance of such a control method in the future.

\section{References}

1) Cosmo, M. L. and Lorenzini, E. C.: Tethers in Space Handbook-Third Edition-Tethers Unlimited, Smithsonian Astrophysical Observatory, NASA Marshall Space Flight Center, Cambridge, 1989.

2) Carrol, J. A.: Tether Applications in Space Transportation, Acta Astronautica, 13 (1986), pp. 165-174.

3) Stuart, D. G.: Guidance and Control for Cooperative Tether-mediated Orbital Rendezvous, J. Guid. Control Dynam., 13 (1990), pp. 1102-1108.

4) Ishige, Y., Kawamoto, S., and Kibe, S.: Study on Electrodynamic Tether System for Space Debris Removal, Acta Astronautica, 55 (2004), pp. 917-929.

5) Netzer, E. and Kane, T. R.: Deployment and Retrieval Optimization of a Tethered Satellite System, J. Guid. Control Dynam., 16 (1993), pp. 1085-1091.

6) Fujii, H. A. and Anazawa, S.: Deployment/retrieval Control of Tethered Subsatellite through an Optimal Path, J. Guid. Control Dynam., 17 (1994), pp. 1292-1298.
7) Fujii, H. A. and Kojima, H.: Optimal Trajectory Analysis for Deployment/Retrieval of Tethered Subsatellite Using Metric, J. Guid. Control Dynam., 26 (2003), pp. 177-179.

8) Steindl, A. and Troger, H.: Optimal Control of Deployment of a Tethered Subsatellite, Nonlinear Dynam., 31 (2003), pp. 257-274.

9) Blanksby, C. and Trivailo, P.: Assessment of Actuation Methods for Manipulating Tip Position of Long Tethers, Space Technol., 20 (2000), pp. 31-39.

10) Williams, P., Blanksby, C., Trivailo, P., and Fujii, H. A.: In-plane Payload Capture Using Tethers, Acta Astronautica, 57 (2005), pp. 772-787.

11) Williams, P. and Blanksby, C.: Prolonged Payload Rendezvous Using a Tether Actuator Mass, J. Spacecraft Rockets, 41 (2004), pp. 889-892.

12) Banerjee, A. K. and Kane, T. R.: Tethered Satellite Retrieval with Thruster Augmented Control, J. Guid. Control Dynam., 7 (1984), pp. 45-50.

13) Pradeep, S. and Kumar, K.: Extension of Tethered Satellites in the Atmosphere, Acta Astronautica, 52 (2003), pp. 1-10.

14) Lorenzini, E. C., Bortolami, S. B., Rupp, C., and Angrilli, F.: Control and Flight Performance of Tethered Satellite Small Expendable Deployment System-II, J. Guid. Control Dynam., 19 (1996), pp. $1148-1156$.

15) Kawasaki, N. and Shimemura, E.: A Method of Designing State Feedback Law to Allocate All Eigenvalues in the Assigned Region by Changing the Imaginary Parts, Trans. Soc. Instrument Control Eng., 16 (1980), pp. 14-20.

16) Kojima, H., Sugimoto, Y., and Furukawa, Y.: Experimental Study on Dynamics and Control of Tethered Satellite Systems with Climber, Acta Astronautica, 69 (2011), pp. 96-108.

17) Kojima, H., Furukawa, Y., and Trivailo, P. M.: Experimental Verification of Periodic Libration of Tethered Satellite System in Elliptic Orbit, 
J. Guid. Control Dynam., 34 (2011), pp. 614-618.

18) Kojima, H., Furukawa, Y., and Trivailo, P. M.: Experimental Study on Delayed Feedback Control for Libration of Tethered Satellite System, J. Guid. Control Dynam., 35 (2012), pp. 998-1002.

19) Liangdong, L. and Bainum, P. M.: Effect of Tether Flexibility on the Tethered Shuttle Subsatellite Stability and Control, J. Guid. Control Dynam., 2 (1989), pp. 866-873.

20) Misra, A. K., Amier, Z., and Modi, V. J.: Attitude Dynamics of Threebody Tethered Systems, Acta Astronautica, 17 (1988), pp. 1059-1068.

\section{Appendix. LQR Design with Pole Assignment Shift ${ }^{15}$}

The following LQR problem is given:

"Find a feedback control gain vector $\boldsymbol{F}$ for the state feedback control: $\boldsymbol{u}=-\boldsymbol{F} \boldsymbol{x}$, such that the following quadratic cost function is minimized:

$$
J=\int_{0}^{\infty}\left(\boldsymbol{x}^{\mathrm{T}} \boldsymbol{Q} \boldsymbol{x}+\boldsymbol{u}^{\mathrm{T}} \boldsymbol{R} \boldsymbol{u}\right) \mathrm{d} t
$$

subject to the dynamic system given by a state space form
$\dot{x}=\boldsymbol{A x}+\boldsymbol{B u}$."

The control gain vector $\boldsymbol{F}$ is given by

$$
\boldsymbol{F}=\boldsymbol{R}^{-1} \boldsymbol{B}^{\mathrm{T}} \boldsymbol{P}
$$

where $\boldsymbol{P}$ is a symmetric positive definite solution of the Riccati equation

$$
\boldsymbol{A}^{\mathrm{T}} \boldsymbol{P}+\boldsymbol{P A}-\boldsymbol{P} \boldsymbol{B} \boldsymbol{R}^{-1} \boldsymbol{B}^{\mathrm{T}} \boldsymbol{P}+\boldsymbol{Q}=0 .
$$

In the pole assignment shift technique, in order to introduce robustness to the controller, the system matrix $\boldsymbol{A}$ in the above Riccati equation is replaced with

$$
\boldsymbol{A}=\boldsymbol{A}+\gamma \boldsymbol{I}, \quad(\gamma>0)
$$

where $\boldsymbol{I}$ is the unit vector.

K. Yamada Associate Editor 